\title{
Epidemic prevention strategies for COVID-19 key populations
}

\author{
Author: \\ Cheng Yuan yuan（程园园） \\ Address for correspondence: \\ E-mail:henuhh@outlook.com
}

Affiliation:

Henan University Huaihe hospital, Kaifeng City, Henan Province, China.

\begin{abstract}
Backgroud: Since 2021, all outbreaks of COVID-19 within mainland China have been associated with imported cases from abroad. The outbreak in Putian, Fujian province, occurred without obvious loopholes in the implementation of epidemic prevention, indicating loopholes in the current epidemic prevention strategies in mainland China. The loopholes should be identified and the epidemic prevention strategies should be improved as soon as possible.

Methods: Is there a need to further extend the medical observation period and community health surveillance period for key populations? Is there a need to increase the number of nucleic acid screenings? Is there a need to change the methods of extraction of nucleic acid screening samples? Is there a need to expand the scope of the population for routine epidemic prevention surveillance? The analysis of these questions would contribute to the improvement of the COVID-19 epidemic prevention strategies.
\end{abstract}

Results: There are larger outbreaks following the current intensive isolation medical observation period and community health surveillance period, the phenomenon shows that the loopholes in the assessment of cross-infection risk and health surveillance in China's current COVID-19 prevention and control strategies, especially during health surveillance after the entry of undetected infected individuals into the community when they emerge during isolation medical observation, and during health surveillance in the community for those who regain positive status after case cure. Conclusions: The key points to improve epidemic prevention strategies includ that a scientific and rational assessment around the mean incubation period, cross-infection risk, and surveillance efficiency of COVID-19, and updating routine epidemic prevention surveillance measures for key populations, recently cured patients of COVID-19, and immersion populations.

Keywords: COVID-19, epidemic prevention strategy, incubation period, cross-infection

Since 2021, all outbreaks of COVID-19 within mainland China have been associated with imported cases from abroad ${ }^{[1]}$. The outbreaks in Nanjing City, Jiangsu province, from July 20 to August 18, $2021^{[2,3]}$, Zhengzhou City, Henan Province, from July 30 to August 28, $2021^{[4-7]}$, and Putian City, Fujian Province, from September 10 to October 7, $2021^{[8-10]}$ The successive outbreaks of COVID-19 were caused by imported cases from abroad. To prevent the risk of 
importation of COVID-19 from outside China, measures related to the prevention and control of the epidemic among inbound persons have been introduced in mainland China, making inbound persons from outside China a priority population for COVID-19 prevention ${ }^{[11,12]}$, and local measures have also been introduced in various provinces and cities.

For example, the epidemic prevention measures in Nanjing City, Jiangsu Province [13, 14]: the implementation of "14+14" health management measures for inbound personnel, that is: 14 days of centralized isolation medical observation period to meet the conditions for release from isolation, continue to strictly implement 14 days of home isolation medical observation. Home isolation medical observation of the second day (after 48 hours), the 14th day to carry out nucleic acid testing, 2 tests are negative, can be included in the regular management. After the release of centralized isolation observation, they should be included in the community grid management, and home observation management until they leave the medium and high risk area for 28 days, and 1 nucleic acid test each on the 3rd and 14th day of home observation. People entering from Nanjing Lukou Airport and port terminals will be sent to isolation hotels for 14 days of centralized isolation medical observation; those destined for Nanjing will continue to be under 14 days of home isolation medical observation after being released from centralized isolation. Shanghai controlled transit back to Nanjing personnel, in all the implementation of the " $3+11$ " centralized isolation, based on the continued implementation of 14 days home isolation. In foreign provinces and cities to enter, the implementation of 14 days of centralized isolation of people returning to Nanjing, you need to continue to implement home isolation to the end of the 28-day period of entry.

Putian City, Fujian Province, epidemic prevention measures ${ }^{[15]}$ : people entering Putian City in the port of entry strictly implement 14 days of centralized medical observation, after the lifting of centralized medical observation, and when the destination of them is Putian City, they would be "point-to-point" pick-up back to the destination to continue to implement 7 days of home health measures, health surveillance after the lifting of the 7-day health follow-up visits.During the period of centralized medical observation, the nucleic acid test of them are conducted at the time of entry, the first day of centralized isolation, the fourth day, the seventh day, the tenth day and the fourteenth day, and the test results are all negative before the release of centralized medical observation; the nucleic acid test is conducted at the first day and the seventh day of home health surveillance, and the test results are all negative before the release of health surveillance; the nucleic acid test is conducted at the seventh day of health follow-up.

Epidemic prevention measures in Zhengzhou City, Henan Province ${ }^{[16]}: 14$ days of isolated medical observation for incoming persons. After release from quarantine, 7 days of home health surveillance should be conducted, during which body temperature and symptoms should be red, mobility should be reduced, personal protection should be done when going out, no gathering activities should be attended, and nucleic acid testing should be conducted once on day 2 and once on day 7. Personnel returned to Zhengzhou should has been isolated for 14 days in the first entry point outside of Zhengzhou, who should be reported to the destination community within 48 hours before arriving in Zhengzhou, arrived in Zhengzhou by the local "point-to-point" closed- 
loop pick-up, the strict implementation of the " $7+2$ " control measures ( 7 days home medical observation, once the Nucleic acid and once serum antibody testing).

However, after strict control, there are still serious outbreaks in some provinces and cities in mainland China. The difference was that Nanjing in Jiangsu and Zhengzhou in Henan caused the outbreak because the relevant personnel failed to strictly implement the existing epidemic prevention measures, and there were negligence and loopholes in the implementation of epidemic prevention, which led to the outbreak and spread of the epidemic. Putian, Fujian Province, however, had an outbreak without obvious loopholes in the implementation of epidemic prevention. The Putian phenomenon shows that there are undetected loopholes in the current COVID-19 prevention and control strategies in mainland China, especially in the assessment of cross-infection risk and community health surveillance.

The loopholes may exist during health surveillance in the community. Involves undetected infected individuals who present during the isolated medical observation period and those who return positive after being cured of COVID-19.These loopholes should be identified immediately and the epidemic prevention strategies should be improved as soon as possible.

The discussion of these questions will be beneficial to the improvement of COVID-19 prevention and control strategies.For exampl, is there a need to further extend the medical observation period and community health surveillance period? Is there a need to increase the number of nucleic acid screenings? Is there a need to change the way nucleic acid screening samples are taken? Is there a need to expand the scope of the population under routine epidemic prevention surveillance?

Question 1: Whether the medical isolation observation period and community health surveillance period for key populations need to be further extended.

Whether to extend the medical isolation observation period and community health surveillance period for key populations should be decided mainly based on the mean incubation period of COVID-19. The great value of incubation period needs to be given some attention, and the great value may be an error value due to the mixture of bias (e.g., recall bias, detection bias), and the great value of incubation period needs to be treated with caution until the bias is excluded. incubation maxima are appropriate as a matter of concern rather than as an immediate basis for adjusting medical observation periods and community health surveillance periods. It has been demonstrated that the suspected source case of the outbreak in Putian, Fujian, in September 2021 who was infected during intensive isolation after entry ${ }^{[17]}$, it suggesting that the incubation period of that case was not 38 days after excluding bias. In the absence of additional evidence of significant changes in the mean incubation period of COVID-19, there is no need to adjust the medical isolation observation period for the focal population to avoid increasing the cost of isolation and the risk of cross-infection ${ }^{[18]}$. However, community health surveillance of priority populations should be integrated into routine epidemiological surveillance (see the discussion in question four for details).

Question 2: Is there a need to increase the number of population-based nucleic acid screenings. 
Increasing the number of nucleic acid screenings in populations (including those within organizations and in regions, such as those within enterprises and institutions, schools, as well as those in communities and administrative districts) has the potential to improve the odds of detecting COVID-19 infections, but it also increases the risk of cross-infection.In July 2021, cross-infection of COVID-19 were occurred in nucleic acid screening sites of which Nanjing City ${ }^{[19]}$ and Yangzhou City ${ }^{[20-28]}$, Jiangsu Province. It had been confirmed that the suspected source case in Putian City, Fujian Province, who was infected during inbound isolation ${ }^{[17]}$, although it has not been confirmed that it was cross-infected while undergoing nucleic acid screening, but this possibility has not been ruled out. Crowding is an important risk factor for cross-infection with COVID-19, and there is reason to believe that cross-infection events resulting from nucleic acid screening activities will still occur. The decision to increase the number of population-based nucleic acid screenings should be made by weighing multiple factors such as the cost-benefit of screening, the average incubation period for COVID-19, the increased odds of finding a COVID-19-positive person, and the additional increased risk of cross-infection, of which the additional increased risk factor of cross-infection should be given sufficient weight.

Question 3: Is there a need to change the sampling method for nucleic acid screening.

The currently implemented sampling method of nasal or pharyngeal swabs taken by medical personnel has the potential for cross-infection and is a serious test of the individual protection and resistance to infection of those to be examined in the field. Mechanical repetitive operations can easily cause fatigue among medical staff, resulting in lower efficiency and consistency of sampling results, and can be accompanied by a series of problems such as higher error rates and higher mixing rates of unqualified samples. A scientific assessment of the potential for crosscontamination and the consistency of sampling results during sampling by medical staff is needed to determine whether to change or supplement the nucleic acid screening sample extraction method. Alternatives to nasal or pharyngeal swab sampling should be evaluated, using selfsampling of mouthwash or saliva ${ }^{[29-42]}$, in which the screened person seals a sample of mouthwash or saliva for self-sampling and submits it to a staff member under the guidance of the staff member to complete the sampling. The staff can be short-trained volunteers and other personnel who can complete the sampling process through remote online guidance, which is an effective solution to both reduce the risk of cross-contamination and alleviate the shortage question of medical staff. Therefore, it is necessary to consider the use of mouthwash or saliva self-sampling as a complementary solution to change the sampling method of nasal or pharyngeal swabs taken exclusively by medical staff.

Question 4: Is there a need to expand the scope of the population for routine epidemic prevention surveillance.

The COVID-19 outbreaks in Putian and other places in Fujian has omitted routine epidemic prevention surveillance of close contacts and sub-close contacts of key populations from the epidemic prevention strategies. Routine epidemic prevention surveillance here refers to routine nucleic acid test screening, epidemic prevention active surveillance, and epidemic prevention 
passive surveillance of the population over a longer period of time (e.g., one month, three months, or longer). The contents of the active and passive surveillance include the temperature (whether fever is present) and symptoms such as cough, weakness, sore throat, dyspnea, loss of taste and smell of the personnel under surveillance, and related symptoms of the close contacts of the personnel under surveillance. Considering the cost of health expenditure and social mobilization, it is difficult to maintain the normality of nucleic acid screening for the whole population in the administrative area, and the implementation of its epidemic prevention active and passive surveillance is even more difficult. Therefore, the population for regular acid test screening and epidemic prevention active and passive surveillance are not the entire population in the administrative area, but a specific population which is defined as the close contacts and sub-close contacts, or called "immersion population", in the circle of daily life of the key population and recently cured COVID-19 patients, who will be included in the routine COVID-19 surveillance with the key population and recently cured COVID-19 patients for a longer period of time (e.g., one month, three months, or longer).This population will be routinely screened for nucleic acid testing and for active and passive surveillance.

The question of the frequency of nucleic acid testing screening for key population, recently cured COVID-19 patients, and the immersion population should still be decided by weighing multiple factors such as the average incubation period of COVID-19, increase the odds of detecting COVID-19-infected patients and additional increased risk of cross-infection (see the discussions in Question 2 for details). The repeatable random sampling should be used with the sampling method for nucleic acid testing screening and epidemic prevention surveillance in those populations, and the sampling rate should be developed scientifically, reasonably, and dynamically adjusted according to the actual situation. This can make the scope of epidemic prevention and surveillance more precise, can significantly save the cost of health expenditure and social mobilization, and is more conducive to the normal operation of epidemic prevention. Therefore, it is necessary to expand the population scope of routine epidemic prevention and surveillance, which is the key to make up for the omission of epidemic prevention strategies.

From October 17, 2021 to 24:00 on November 5, a total of 918 infections were reported in China, affecting 44 cities in 20 provinces (Shanxi, Hubei, Hunan, Zhejiang, Jiangsu, Inner Mongolia, Beijing, Guizhou, Shandong, Gansu, Qinghai, Ningxia, Yunnan, Heilongjiang, Hebei, Henan, Jiangxi, Sichuan, Chongqing, and Liaoning), and the results of viral gene sequencing and flow transfer traceability showed that the outbreaks, including the current round of the epidemic of Ejina Banner, Inner Mongolia was caused by multiple unrelated foreign import sources ${ }^{[43]}$, and analysis of this situation shows that when there are undetected foreign import cases or asymptomatic infected persons in border cities and port cities, the involvement of foreign tourists and the movement of people across provinces and cities ignite the epidemic and intensify its spread. At this time, a buffer mechanism between border cities and port cities and non-border cities and non-port cities should be established, including setting the necessary buffer time and buffer space, and using the buffer mechanism to slow down the expansion of the epidemic and prevent the epidemic from penetrating the epidemic prevention front of more cities in a short period of time through convenient transportation channels. 
The key points to improve epidemic prevention strategies includs that a scientific and rational assessment around the mean incubation period, cross-infection risk, and surveillance efficiency of COVID-19, and updating routine epidemic prevention surveillance measures for key populations, recently cured patients of COVID-19, and immersion populations, and the establishment of necessary buffer mechanisms between cities.

References.

[1] China Health and Welfare Commission Epidemic Bulletin [EB/OL]. http://www.nhc.gov.cn/xcs/yqtb/list_gzbd_2.shtml. [2021-10-12].

[2] Briefing on the positive detection of COVID-19 at Nanjing Lukou International Airport [EB/OL]. http://wjw.nanjing.gov.cn/njswshjhsywyh/202107/t20210721_3080544.html. [2021-07-21]

[3] Nanjing Municipal People's Government Information Office held the 30th Nanjing COVID-19 Epidemic Prevention and Control Press Conference Nanjing's entire area turned into a low-risk area [EB/OL]. http://www.nanjing.gov.cn/njxx/202108/t20210820_3107402.html.[2021-08-20]

[4] Update on the COVID-19 outbreak in Henan Province as of 24:00 July 30, 2021 [EB/OL]. http://wsjkw.henan.gov.cn/2021/07-31/2193583.html. [2021-07-31].

[5] Zhengzhou City COVID-19 Epidemic Prevention and Control Leading Group Office issued Circular No. 4 [EB/OL]. https://www.henan.gov.cn/2021/08-24/2299862.html. [2021-08-23].

[6] Zhengzhou City COVID-19 Epidemic Prevention and Control Leading Group Office issued Circular No. 5 [EB/OL]. https://www.henan.gov.cn/2021/08-

24/2299861.html.2021-08-23]

[7] Zhengzhou City COVID-19 Epidemic Prevention and Control Leading Group Office issued Circular 35 [EB/OL]. https://www.henan.gov.cn/2021/08-30/2302379.html.202108-30]

[8] Putian City held a press conference on the prevention and control of the epidemic [EB/OL]. http://www.putian.gov.cn/ztzl/tcyqfkhjjshfz/yqdt/qptsyqdt/202109/t20210910_1646470.h tm. [2021-09-10]

[9] Putian City COVID-19 Epidemic Situation [EB/OL]. http://www.putian.gov.cn/ztzl/tcyqfkhjjshfz/yqdt/qptsyqdt/202109/t20210911_1646499.h tm.[2021-09-11]

[10] Putian City Notice on Adjusting the Risk Level of the Epidemic (No. 36) [EB/OL]. http://www.putian.gov.cn/ztzl/tcyqfkhjjshfz/tzgg/202110/t20211007_1659605.htm.[2021 $-10-07]$

[11] Summary of policy measures for epidemic prevention and control across China [EB/OL]. http://www.gov.cn/zhuanti/2021yqfkgdzc/index.htm.[2021-10-9] 
[12] COVID-19 Prevention and Control Program (8th Edition) [EB/OL]. https://oss.henan.gov.cn/typtfile/20210825/01bf2af26d1543a694203718d06e197f.pdf.[20 21-08-11]

[13] Jiangsu Province to implement graded management of people coming to Suzhou during the Spring Festival [EB/OL]. https://www.jiangsu.gov.cn/art/2021/1/20/art_83409_9989550.html.[2021-01-20]

[14] Briefing on the ten gates to do a good job related to the prevention and control of winter and spring epidemics in Nanjing [EB/OL]. http://nanjing.gov.cn/zdgk/202101/t20210126_2804094.html.[2021-01-14]

[15] Fujian Province travel epidemic prevention policy measures [EB/OL]. http://www.fujian.gov.cn/zwgk/ztzl/yqfk/fkzc/fkzc/202109/t20210915_5688897.htm.[20 21-09-15]

[16] Zhengzhou City COVID-19 Epidemic Prevention and Control Leading Group Office issued Circular No. 2 [EB/OL]. https://www.henan.gov.cn/2021/0824/2299883.html.[2021-08-23]

[17] CDC: Putian outbreak suspected source or infected during isolation [EB/OL]. http://news.cctv.com/2021/09/16/ARTIvlfyIfncRGqgUHPMlywa210916.shtml.2021-0916]

[18] L. Grout, A. Katar, D. Ait Ouakrim, J.A. Summers, A. Kvalsvig, M.G. Baker, T. Blakely, N. Wilson, Failures of quarantine systems for preventing COVID -19 outbreaks in Australia and New Zealand, Medical Journal of Australia, 215 (2021) 320-324.

[19] New confirmed cases of COVID-19 in Nanjing from 0:00 to 24:00 on July 31 [EB/OL]. http://wjw.nanjing.gov.cn/njswshjhsywyh/202108/t20210801_3089858.html.2021-08-01]

[20] Epidemic notification: 26 new confirmed cases of COVID-19 in Yangzhou [EB/OL].http://wjw.yangzhou.gov.cn/yzwshjh/ywkd/202108/9a16adef18e3488db64a3eb 41314e262.shtml. [2021-08-02].

[21] Epidemic notification: 40 new confirmed cases of COVID-19 in Yangzhou City [EB/OL]. http://wjw.yangzhou.gov.cn/yzwshjh/ywkd/202108/fa41d8efb3644fd68393c0aed478f03e .shtml.[2021-08-03].

[22] Epidemic notification: 32 new confirmed cases of COVID-19 in Yangzhou City [EB/OL]. http://wjw.yangzhou.gov.cn/yzwshjh/ywkd/202108/d62c0e11801f4012b58774ff64b89ba c.shtml.[2021-08-04]

[23] Epidemic notification: 36 new confirmed cases of COVID-19 in Yangzhou City [EB/OL]. http://wjw.yangzhou.gov.cn/yzwshjh/ywkd/202108/f624c59b2d484313bad13aa5a4bd9be 0.shtml.[2021-08-05] 
[24] Epidemic notification: 58 new confirmed cases of COVID-19 in Yangzhou City [EB/OL].

http://wjw.yangzhou.gov.cn/yzwshjh/ywkd/202108/073cd0cd0856473a943636e9a33e80b e.shtml.[2021-08-06]

[25] Epidemic notification: 52 new confirmed cases of COVID-19 in Yangzhou City. [EB/OL].http://wjw.yangzhou.gov.cn/yzwshjh/ywkd/202108/b539c3eb401a4e148bd588b 7afc12475.shtml. [2021-08-07]

[26] Epidemic notification: 36 new confirmed cases of COVID-19 in Yangzhou City [EB/OL]. http://wjw.yangzhou.gov.cn/yzwshjh/ywkd/202108/c60718f241a0474986e20bcac66def7 b.shtml.[2021-08-08 ]

[27] Epidemic notification: 38 new confirmed cases of COVID-19 in Yangzhou [EB/OL]. http://wjw.yangzhou.gov.cn/yzwshjh/ywkd/202108/ff6674b99aa264d37814af7d34b84b59 6.shtml.[2021-08-09]

[28] Epidemic notification: 48 new confirmed cases of COVID-19 in Yangzhou City [EB/OL].http://wjw.yangzhou.gov.cn/yzwshjh/ywkd/202108/a9fa72edc27e435abad92ce9 a1d77955.shtml.[2021-08-10 ]

[29] Wyllie A L, Fournier J, Casanovas-Massana A, et al. Saliva or nasopharyngeal swab specimens for detection of SARS-CoV-2[J]. New England Journal of Medicine, 2020, 383(13): 1283-1286.

[30] Iwasaki S, Fujisawa S, Nakakubo S, et al. Comparison of SARS-CoV-2 detection in nasopharyngeal swab and saliva[J]. Journal of Infection, 2020, 81(2): e145-e147.

[31] Mittal A, Gupta A, Kumar S, et al. Gargle lavage as a viable alternative to swab for detection of SARS-CoV-2[J]. The Indian journal of medical research, 2020, 152(1-2): $77-81$.

[32] Bennett S, MacLean A, Gunson R. Verification of Cepheid Xpert Xpress Flu/RSV assay for use with gargle samples, sputa and endotracheal secretions[J]. Journal of Hospital Infection, 2019, 101(1): 114-115.

[33] Bennett S, Davidson R S, Gunson R N. Comparison of gargle samples and throat swab samples for the detection of respiratory pathogens[J]. Journal of virological methods, 2017, 248: 83-86.

[34] Azzi L, Carcano G, Gianfagna F, et al. Saliva is a reliable tool to detect SARS-CoV-2[J]. Journal of Infection, 2020, 81(1): e45-e50.

[35] Mestdagh P, Gillard M, Dhillon S K, et al. Evaluating Diagnostic Accuracy of Saliva Sampling Methods for Severe Acute Respiratory Syndrome Coronavirus 2 Reveals Differential Sensitivity and Association with Viral Load[J]. The Journal of Molecular Diagnostics, 2021, 23(10): 1249-1258. 
[36] Lai C K C, Lui G C Y, Chen Z, et al. Comparison of self-collected mouth gargle with deep-throat saliva samples for the diagnosis of COVID-19: Mouth gargle for diagnosis of COVID-19[J]. Journal of Infection, 2021, 83(4): 496-522.

[37] Considerations for the use of saliva as sample material for COVID-19 testing[EB/OL].https://www.ecdc.europa.eu/en/publications-data/considerations-usesaliva-sample-material-covid-19-testing.[2021-10-20]

[38] Saito M, Adachi E, Yamayoshi S, et al. Gargle lavage as a safe and sensitive alternative to swab samples to diagnose COVID-19: a case report in Japan[J]. Clinical Infectious Diseases, 2020, 71(15): 893-894.

[39] Malecki M, Lüsebrink J, Teves S, et al. Pharynx gargle samples are suitable for SARSCoV-2 diagnostic use and save personal protective equipment and swabs[J]. Infection Control \& Hospital Epidemiology, 2021, 42(2): 248-249.

[40] Goldfarb D M, Tilley P, Al-Rawahi G N, et al. Self-Collected Saline Gargle Samples as an Alternative to Health Care Worker-Collected Nasopharyngeal Swabs for COVID-19 Diagnosis in Outpatients[J]. Journal of clinical microbiology, 2021, 59(4): e02427-20.

[41] Rao M, Rashid F A, Sabri F S A H, et al. Comparing nasopharyngeal swab and early morning saliva for the identification of severe acute respiratory syndrome coronavirus 2 (SARS-CoV-2)[J]. Clinical Infectious Diseases, 2021, 72(9): e352-e356.

[42] Procop G W, Shrestha N K, Vogel S, et al. A direct comparison of enhanced saliva to nasopharyngeal swab for the detection of SARS-CoV-2 in symptomatic patients[J]. Journal of clinical microbiology, 2020, 58(11): e01946-20.

[43] Transcript of China State Council Joint Prevention and Control Mechanism Press Conference on November 6, 2021 [EB/OL]. http://www.nhc.gov.cn/xcs/fkdt/202111/28aeb8577b8747ed877f582d50f51035.shtml. [2021-11-06 ] 


\section{Supplementaries}

\section{Competing Interest Statement}

The authors declare no competing interest.

\section{Clinical Trial}

NCT12345678

\section{Funding Statement}

There is no external funding, and there is no conflict of interests.

\section{Authors Declaration}

All relevant ethical guidelines have been followed; any necessary IRB and/or ethics committee approvals have been obtained and details of the IRB/oversight body are included in the manuscript.

Yes

All necessary patient/participant consent has been obtained and the appropriate institutional forms have been archived.

Yes

I understand that all clinical trials and any other prospective interventional studies must be registered with an ICMJE-approved registry, such as ClinicalTrials.gov. I confirm that any such study reported in the manuscript has been registered and the trial registration ID is provided (note: if posting a prospective study registered retrospectively, please provide a statement in the trial ID field explaining why the study was not registered in advance). 
I have followed all appropriate research reporting guidelines and uploaded the relevant EQUATOR Network research reporting checklist(s) and other pertinent material as supplementary files, if applicable.

Yes

Data Availability Statement:

All data, models, and code generated or used during the study appear in the submitted article. 